\title{
44581 - IDENTIFICATION OF GENETIC RISK FACTORS PREDICTIVE OF ADVERSE OUTCOMES AFTER CARDIAC SURGERY
}

Disclosure: Grants/research support - Heart \& Stroke Foundation of Ontario

\author{
Natasha Kravea, Toronto General Hospital, University Health Network, Toronto, ON, \\ Canada; \\ Ludwik Fedorko, Toronto General Hospital, University Health Network; \\ W Scott Beattie, Toronto General Hospital, University Health Network; \\ Wanda Frodis, Toronto General Hospital, University Health Network; \\ J Carroll, Djaiani; \\ H Poonawala, Toronto General Hospital, University Health Network; \\ G Djaiani, Toronto General Hospital, University Health Network;
}

INTRODUCTION:Major surgical procedures in elderly and high cardiovascular risk population are still associated with relatively high incidence of morbidity and mortality. Thrombotic events either in form of embolic events or cardiac ischemia constitute significant proportion of overall morbidity. Detailed knowledge of common genetic variants affecting clotting cascade which may contribute to the adverse events during surgical stress, is still lacking. Association mapping studies have played an important role in the identification of numerous susceptibility loci for a wide range of common complex diseases1. The International HapMap Project, an important source of validated single nucleotide polymorphisms (SNPs) and detailed data about haplotype structure across extended genomic regions in several populations, has provided the most comprehensive resources for the design of SNP-based association studies2. The aim of this study was, based on the HapMap data, to create an efficient tool for the identification of SNPs and/or haplotypes conferring a higher risk of adverse outcomes after cardiac surgery.

METHODS:Local REB approval was obtained for this study. Following REB approval,310 Caucasian and 56 Asian cardiac surgery patients with well-defined demographical and surgical characteristics were included in this study. The list of chosen candidate genes included ACE, GP1BA, F2, F2R, F3, F5, F7, F13A1, FBG, PAI-1, TFPI, TAFI, MTHFR, CRP, IL6, ADRB1, ADRB2 and ADRA2A. To cover genetic variation across each of these genes we genotyped altogether 156 SNPs. A set of 50 unlinked markers, chosen from ALFRED database, was genotyped and used to control for population stratification using software STRUCTURE. Allele frequencies were estimated using gene counting. Haplotypes were inferred with the software PHASE.

RESULTS:We performed genotyping and estimated allele frequencies of a panel 156 SNPs that would represent genetic variation across 18 genes involved in hemostasis in two ethnic groups of cardiac surgery patients. Most of the SNPs were in Hardy-Weinberg equilibrium. We inferred haplotypes and estimated haplotype distribution across candidate gene regions.

CONCLUSIONS:Using the HapMap data, we have compiled a panel of SNPs representing common variation across 18 candidate genes involved in hemostasis. Extensive bioinformatic analysis of chosen SNPs has allowed us to design a scalable genotyping assay based on the 
Sequenom MassArray platform that was successfully applied for genotyping of a comparatively small cohort of Canadian cardiac surgery patients.

This assay could later be modified to accommodate the sample size requirements for association study. This assay format provides a tool for studies of multilocus genetic risk factors in large cardiovascular disease cohorts, and for subsequent development of diagnostic tests for preoperational evaluation risk of bleeding and possibly other adverse outcomes during cardiac surgery.

REFERENCES: 1.Nature 2004 429:446-452,2.Nat Genet 2006 38:1251-1260 